\title{
SOME ASYMPTOTIC SPECTRAL FORMULAE FOR THE EIGENVALUES OF THE LAPLACIAN
}

\author{
E. M. E. ZAYED ${ }^{1}$
}

(Received 30 June 1987; revised 15 December 1987)

\begin{abstract}
In this paper we shall derive some asymptotic formulae for spectra of the third boundary value problem in $R^{n}, n=2$ or 3 , linked with variation of a positive function entering the boundary conditions. Further results may be obtained.
\end{abstract}

\section{Introduction}

The underlying problem is to deduce the precise shape of a membrane from the complete knowledge of the eigenvalues $\mu_{k}(\sigma)$ for the Laplace operator $\Delta_{n}$ in $R^{n}, n=2$ or 3 .

Let $\Omega$ be a simply connected bounded domain in $R^{n}$ with a smooth boundary $\partial \Omega$ in the case $n=2$, or a smooth bounding surface $S$ in the case $n=3$. Consider the third boundary value problem

$$
\left.\left(\Delta_{n}+\lambda\right) u=0 \text { in } \Omega, \quad(\partial / \partial n+\sigma) u=0 \quad \text { on } \partial \Omega \text { (or } S\right),
$$

where $\partial / \partial n$ denotes differentiation along the inward-pointing normal to $\partial \Omega$ (or $S$ ), and $\sigma$ is a positive function. Denote its eigenvalues, counted according to multiplicity, by

$$
0<\mu_{1}(\sigma) \leq \mu_{2}(\sigma) \leq \cdots \leq \mu_{k}(\sigma) \leq \cdots \rightarrow \infty \quad \text { as } k \rightarrow \infty .
$$

At the beginning of this century, the principal problem was that of investigating the asymptotic distribution of the eigenvalues (1.2). It is well known (see

\footnotetext{
${ }^{1}$ Mathematics Department, Zagazig University Faculty of Science, Zagazig, Egypt Present address: Mathematics Department, Faculty of Science, University of Emirates, P.O. Box 15551 Al Ain, Abu Dhabi, United Arab Emirates.

(C) Copyright Australian Mathematical Society 1988, Serial-fee code 0334-2700/88
} 
[1]) that, in the case $n=2$,

$$
\mu_{k}(\sigma) \sim(4 \pi /|\Omega|) k \quad \text { as } k \rightarrow \infty,
$$

while, in the case $n=3$,

$$
\left.\mu_{k}(\sigma) \sim\left(6 \pi^{2} / V\right) k\right)^{2 / 3} \quad \text { as } k \rightarrow \infty,
$$

where $|\Omega|$ and $V$ respectively are the area and the volume of the domain $\Omega$.

The problem of determining further information about the geometry of $\Omega$ as well as the impedance $\sigma$ from a complete knowledge of the eigenvalues $\mu_{k}(\sigma)$ has been discussed recently in [6] when $n=2$ and in [8] when $n=3$, in the case $\sigma=$ constant $>0$, through the asymptotic expansions of the spectral function

$$
\theta(t)=\sum_{k=1}^{\infty} \exp \left\{-t \mu_{k}(\sigma)\right\}
$$

for small positive $t$.

Thus in the case $n=2,0<\sigma \ll 1$

$$
\theta(t)=\frac{|\Omega|}{4 \pi t}+\frac{|\partial \Omega|}{8(\pi t)^{1 / 2}}+\frac{1}{6}\left(1-\frac{3 \sigma}{\pi}|\partial \Omega|\right)+O\left(t^{1 / 2}\right) \quad \text { as } t \rightarrow 0,
$$

while in the case $n=3,0<\sigma \ll 1$

$$
\theta(t)=\frac{V}{(4 \pi t)^{3 / 2}}+\frac{S}{16 \pi t}+\frac{1}{12 \pi^{3 / 2} t^{1 / 2}} \int_{S}(H-3 \sigma) d S+O\left(t^{1 / 2}\right) \quad \text { as } t \rightarrow 0,
$$

where $H=1 / 2\left(1 / R_{1}+1 / R_{2}\right), R_{1}$ and $R_{2}$ are the principal radii of curvature.

With reference to Section 11 of [6], (1.6) may be interpreted as:

(i) $\Omega \subseteq R^{2}$ is a convex domain and we have the impedance boundary condition of (1.1) with small impedance $\sigma$, or

(ii) $\Omega \subseteq R^{2}$ is a convex domain with $3 \sigma / \pi|\partial \Omega|$ holes and has area $|\Omega|$ and its boundary has length $|\partial \Omega|$ together with the Neumann boundary condition, provided $3 \sigma / \pi|\partial \Omega|$ is an integer.

Similarly, with reference to Section 11 of [8], (1.7) may be interpreted as:

(i) $\Omega \subseteq R^{3}$ is a convex domain and we have the impedance boundary condition of (1.1) with small impedance $\sigma$, or

(ii) $\Omega \subseteq R^{3}$ is a convex domain, has volume $V$ and its surface $S$ has the mean curvature $(H-3 \sigma)$ together with the Neumann boundary condition.

In Theorem 1, we generalise the results (1.6) and (1.7) to the case when $\sigma$ is a positive function satisfying the Lipschitz condition, by using the expression

$$
\sum_{k=1}^{\infty}\left\{\mu_{k}(\sigma)+P\right\}^{-2},
$$

where $P$ is a positive constant. 
In Theorem 2, we show that this generalisation plays an important role in establishing a method to study the asymptotic behaviour of the difference

$$
\sum_{\mu_{k}(\sigma) \leq \lambda}\left\{\mu_{k}\left(\sigma_{2}\right)-\mu_{k}\left(\sigma_{1}\right)\right\}
$$

for large values of $\lambda$, where $\sigma(Q), \sigma_{1}(Q)$ and $\sigma_{2}(Q), Q \in \partial \Omega$ (or $Q \in S$ ) are three distinct functions satisfying the Lipschitz condition, and the summation is taken over all values of $k$ for which $\mu_{k}(\sigma) \leq \lambda$. The method uses an interesting and important Tauberian theorem due to Hardy and Littlewood and developed by Titchmarsh [7].

Theorems 3 and 4 contain further results which can be considered as a generalisation of the results of Theorem 2 .

\section{Statement and proofs of results}

THEOREM 1. If the function $\sigma(Q)$ satisfies the Lipschitz condition and if $P$ is a positive constant, then in the case $n=2$,

$$
\begin{gathered}
\sum_{k=1}^{\infty}\left\{\mu_{k}(\sigma)+P\right\}^{-2}=\frac{|\Omega|}{4 \pi P}+\frac{|\partial \Omega|}{16 P^{3 / 2}}+\frac{1}{6 P^{2}}\left(1-\frac{3}{\pi} \int_{\partial \Omega} \sigma(Q) d Q\right)+O\left(\frac{1}{P^{5 / 2}}\right) \\
\text { as } P \rightarrow \infty, \quad(2.1)
\end{gathered}
$$

while, in the case $n=3$,

$$
\begin{array}{r}
\sum_{k=1}^{\infty}\left\{\mu_{k}(\sigma)+P\right\}^{-2}=\frac{V}{8 \pi P^{1 / 2}}+\frac{S}{16 \pi P}+\frac{1}{24 \pi P^{3 / 2}} \int_{S}\{H(Q)-3 \sigma(Q)\} d Q+O\left(\frac{1}{P^{2}}\right) \\
\text { as } P \rightarrow \infty
\end{array}
$$

Note that the expression (1.8) is just the Laplace tranform of the function $t \theta(t)$ with respect to $t$, and $P>0$ is the Laplace transform parameter. With this connection we deduce that the formulae (2.1) and (2.2) can be considered as a generalisation of the formulae (1.6) and (1.7) respectively.

Note also that Eastham [2] had a similar expression to (1.8), where the eigenvalue parameter appears in the boundary condition.

THEOREM 2. If $\sigma(Q), \sigma_{1}(Q)$ and $\sigma_{2}(Q)$ are three distinct functions satisfying the Lipschitz condition, then in the case $n=2$,

$$
\sum_{\mu_{k}(\sigma) \leq \lambda}\left\{\mu_{k}\left(\sigma_{2}\right)-\mu_{k}\left(\sigma_{1}\right)\right\}=\frac{\lambda}{2 \pi} \int_{\partial n}\left\{\sigma_{2}(Q)-\sigma_{1}(Q)\right\} d Q+o\left(\lambda^{3 / 2}\right) \quad \text { as } \lambda \rightarrow \infty
$$


while, in the case $n=3$,

$\sum_{\mu_{k}(\sigma) \leq \lambda}\left\{\mu_{k}\left(\sigma_{2}\right)-\mu_{k}\left(\sigma_{1}\right)\right\}=\frac{\lambda^{3 / 2}}{3 \pi^{2}} \int_{S}\left\{\sigma_{2}(Q)-\sigma_{1}(Q)\right\} d Q+o\left(\lambda^{2}\right) \quad$ as $\lambda \rightarrow \infty$

Formulae (2.3) and (2.4) can be considered as a generalisation of the familiar formulae of Gel'fand and Levitan [3] for the difference of traces of two SturmLiouville operators.

ProOF OF THEOREM 1. Let $G\left(M, M_{1} ; t\right)$ be the Green's function for the heat equation

$$
\left(\Delta_{n}-\partial / \partial t\right) u=0
$$

subject to the third boundary condition of (1.1) and the initial condition $G\left(M, M_{1} ; t\right) \rightarrow \delta\left(M-M_{1}\right)$ as $t \rightarrow 0$, where $\delta\left(M-M_{1}\right)$ is the Dirac delta function located at the source point $M=M_{1}$. The points $M$ and $M_{1}$ belong to the domain $\Omega$.

Let us write

$$
G\left(M, M_{1} ; t\right)=G_{0}\left(M, M_{1} ; t\right)-g\left(M, M_{1} ; t\right)
$$

where

$$
G_{0}\left(M, M_{1} ; t\right)=(4 \pi t)^{-n / 2} \exp \left(-\left|M-M_{1}\right|^{2} /(4 t)\right),
$$

is the "fundamental solution" of the heat equation (2.5), while $g\left(M, M_{1} ; t\right)$ is the "regular solution" chosen in such a way that $G\left(M, M_{1} ; t\right)$ satisfies the third boundary condition of (1.1).

In what follows, we shall use Laplace transforms with respect to $t$, and use $s^{2}$ as the Laplace transform parameter; thus we define

$$
\bar{G}\left(M, M_{1} ;-s^{2}\right)=\int_{0}^{\infty} e^{-s^{2} t} G\left(M, M_{1} ; t\right) d t .
$$

An application of the Laplace transform to the heat equation (2.5) shows that $\bar{G}\left(M, M_{1} ;-s^{2}\right)$ satisfies the membrane equation

$$
\left(\Delta_{n}-s^{2}\right) \bar{G}\left(M, M_{1} ;-s^{2}\right)=-\delta\left(M-M_{1}\right) \text { in } \Omega,
$$

together with the third boundary condition of (1.1). Consequently, if $n=2$ we deduce that

$$
\bar{G}\left(M, M_{1} ;-s^{2}\right)=(1 / 2 \pi) K_{0}\left(s\left|M-M_{1}\right|\right)-\bar{g}\left(M, M_{1} ;-s^{2}\right),
$$

where $K_{0}$ is the modified Bessel function of the second kind and of zero order. As $M_{1} \rightarrow M$ the equality

$$
\bar{G}\left(M, M_{1} ;-s_{1}^{2}\right)-\bar{G}\left(M, M_{1} ;-s^{2}\right)=\left(s^{2}-s_{1}^{2}\right) \sum_{k=1}^{\infty} \frac{\phi_{k}\left(M_{1}\right) \phi_{k}(M)}{\left\{\mu_{k}(\sigma)+s_{1}^{2}\right\}\left\{\mu_{k}(\sigma)+s^{2}\right\}},
$$


where $\left\{\phi_{k}(M)\right\}$ are normalised eigenfunctions, implies

$$
\begin{aligned}
(1 / 2 \pi) \log & \left(\frac{s_{1}}{s}\right)+\bar{g}\left(M, M ;-s_{1}^{2}\right)-\bar{g}\left(M, M ;-s^{2}\right) \\
& =\left(s_{1}^{2}-s^{2}\right) \sum_{k=1}^{\infty} \frac{\phi_{k}^{2}(M)}{\left\{\mu_{k}(\sigma)+s_{1}^{2}\right\}\left\{\mu_{k}(\sigma)+s^{2}\right\}}
\end{aligned}
$$

(see, for example [4]). Thus we get the formula

$$
\sum_{k=1}^{\infty}\left\{\mu_{k}(\sigma)+s^{2}\right\}^{-2}=|\Omega| /\left(4 \pi s^{2}\right)+(1 / 2 s) \iint_{\Omega} \bar{g}_{s}^{\prime}\left(M, M ;-s^{2}\right) d M
$$

Using methods similar to those used in [6], we can show that

$$
\iint_{\Omega} \bar{g}_{s}^{\prime}\left(M, M ;-s^{2}\right) d M=\frac{|\partial \Omega|}{8 s^{2}}+\frac{1}{3 s^{3}}\left(1-\frac{3}{\pi} \int_{\partial \Omega} \sigma(Q) d Q\right)+O\left(\frac{1}{s^{4}}\right)
$$

On inserting (2.14) into (2.13) and letting $s^{2}=P$, we arrive at the formula (2.1). Similarly, if $n=3$ we deduce that

$$
\bar{G}\left(M, M_{1} ;-s^{2}\right)=\exp \left(-s\left|M-M_{1}\right|\right) /\left(4 \pi\left|M-M_{1}\right|\right)-\bar{g}\left(M, M_{1} ;-s^{2}\right) .
$$

As $M_{1} \rightarrow M$, the equality (2.11) implies

$$
\begin{aligned}
\frac{\left(s_{1}-s\right)}{4 \pi} & +\bar{g}\left(M, M ;-s_{1}^{2}\right)-\bar{g}\left(M, M ;-s^{2}\right) \\
& =\left(s_{1}^{2}-s^{2}\right) \sum_{k=1}^{\infty} \frac{\phi_{k}^{2}(M)}{\left\{\mu_{k}(\sigma)+s_{1}^{2}\right\}\left\{\mu_{k}(\sigma)+s^{2}\right\}},
\end{aligned}
$$

(see, for example [5]). Thus we get the formula

$$
\sum_{k=1}^{\infty}\left\{\mu_{k}(\sigma)+s^{2}\right\}^{-2}=V /(8 \pi s)+(1 /(2 s)) \iiint_{\Omega} \bar{g}_{s}^{\prime}\left(M, M ;-s^{2}\right) d M .
$$

Using methods similar to those used in [8] we can show that

$$
\begin{aligned}
\iiint_{\Omega} \bar{g}_{s}^{\prime}\left(M, M ;-s^{2}\right) d M & \\
=S /(8 \pi s)+\left(1 /\left(12 \pi s^{2}\right)\right) \int_{S}\{H(Q)-3 \sigma(Q)\} d Q+O\left(\frac{1}{s^{3}}\right) & \text { as } s \rightarrow \infty .
\end{aligned}
$$

On inserting (2.18) into (2.17) and letting $s^{2}=P$, we arrive at the formula (2.2). Note that the proof of either (2.14) or (2.18) is omitted here since it is very similar to those obtained in [6] or [8] respectively. 
PROOF OF THEOREM 2. First we derive (2.3) as follows: Let us assume that $\sigma_{2}(Q) \geq \sigma_{1}(Q),(Q \in \partial \Omega)$ and introduce the non-negative and non-decreasing function

$$
\Phi(\lambda)=\sum_{\mu_{k}\left(\sigma_{2}\right) \leq \lambda}\left\{\mu_{k}\left(\sigma_{2}\right)-\mu_{k}\left(\sigma_{1}\right)\right\}
$$

moreover we let

$$
\psi(p)=\sum_{k=1}^{\infty} \frac{\left\{\mu_{k}\left(\sigma_{2}\right)-\mu_{k}\left(\sigma_{1}\right)\right\}^{2}\left\{\mu_{k}\left(\sigma_{2}\right)+2 \mu_{k}\left(\sigma_{1}\right)+3 P\right\}}{\left\{\mu_{k}\left(\sigma_{2}\right)+P\right\}^{3}\left\{\mu_{k}\left(\sigma_{1}\right)+P\right\}} .
$$

Using formula (2.1) first for the function $\sigma_{1}(Q)$, then for the function $\sigma_{2}(Q)$ and subtracting the second one from the first, we find after some reduction that

$$
\begin{array}{r}
2 \sum_{k=1}^{\infty} \frac{\left[\mu_{k}\left(\sigma_{2}\right)-\mu_{k}\left(\sigma_{1}\right)\right]}{\left[\mu_{k}\left(\sigma_{2}\right)+P\right]^{3}}+\psi(P)=\frac{1}{2 \pi P^{2}} \int_{\partial \Omega}\left\{\sigma_{2}(Q)-\sigma_{1}(Q)\right\} d Q+O\left(\frac{1}{P^{5 / 2}}\right) \\
\text { as } P \rightarrow \infty,
\end{array}
$$

which can be rewritten for any $a<\mu_{1}\left(\sigma_{2}\right)$ in the equivalent form

$$
2 \int_{a}^{+\infty} \frac{d \Phi(\lambda)}{(\lambda+P)^{3}}+\psi(P)=\frac{1}{2 \pi P^{2}} \int_{\partial \Omega}\left\{\sigma_{2}(Q)-\sigma_{1}(Q)\right\} d Q+O\left(\frac{1}{P^{5 / 2}}\right)
$$

Further, noting that $\psi(P)=o\left\{\int_{a}^{+\infty}(\lambda+P)^{-3} d \Phi(\lambda)\right\}$ when $P \rightarrow \infty$, we get

$$
\int_{a}^{+\infty} \frac{d \Phi(\lambda)}{(\lambda+P)^{3}} \sim \frac{1}{4 \pi P^{2}} \int_{\partial \Omega}\left\{\sigma_{2}(Q)-\sigma_{1}(Q)\right\} d Q \quad \text { as } P \rightarrow \infty .
$$

Applying a Tauberian theorem of Hardy and Littlewood (see, for example [7], page 364), we find that

$$
\Phi(\lambda) \sim(\lambda /(2 \pi)) \int_{\partial \Omega}\left\{\sigma_{2}(Q)-\sigma_{1}(Q)\right\} d Q \quad \text { as } \lambda \rightarrow \infty .
$$

Analogously, one establishes the asymptotic formula

$$
\sum_{\mu_{k}\left(\sigma_{1}\right) \leq \lambda}\left\{\mu_{k}\left(\sigma_{2}\right)-\mu_{k}\left(\sigma_{1}\right)\right\} \sim(\lambda /(2 \pi)) \int_{\partial \Omega}\left\{\sigma_{2}(Q)-\sigma_{1}(Q)\right\} d Q
$$

$$
\text { as } \lambda \rightarrow \infty \text {. }
$$

Further, noting that

$$
\begin{aligned}
\sum_{\mu_{k}\left(\sigma^{*}\right) \leq \lambda}\left\{\mu_{k}\left(\sigma_{2}\right)-\mu_{k}\left(\sigma_{1}\right)\right\} & \leq \sum_{\mu_{k}(\sigma) \leq \lambda}\left\{\mu_{k}\left(\sigma_{2}\right)-\mu_{k}\left(\sigma_{1}\right)\right\} \\
& \leq \sum_{\mu_{k}\left(\sigma_{*}\right) \leq \lambda}\left\{\mu_{k}\left(\sigma_{2}\right)-\mu_{k}\left(\sigma_{1}\right)\right\}
\end{aligned}
$$


where $\sigma^{*}(Q)=\max \left\{\sigma(Q), \sigma_{2}(Q)\right\}, \sigma_{*}(Q)=\min \left\{\sigma(Q), \sigma_{1}(Q)\right\}$, and the fact that as $\lambda \rightarrow \infty$ the functions:

$$
\begin{aligned}
\sum_{\mu_{k}\left(\sigma^{*}\right) \leq \lambda} & \left\{\mu_{k}\left(\sigma_{2}\right)-\mu_{k}\left(\sigma_{1}\right)\right\} \\
& =\sum_{\mu_{k}\left(\sigma^{*}\right) \leq \lambda}\left\{\mu_{k}\left(\sigma^{*}\right)-\mu_{k}\left(\sigma_{1}\right)\right\}-\sum_{\mu_{k}\left(\sigma^{*}\right) \leq \lambda}\left\{\mu_{k}\left(\sigma^{*}\right)-\mu_{k}\left(\sigma_{2}\right)\right\},
\end{aligned}
$$

and likewise for $\sigma_{*}$ are asymptotically equal to

$$
(\lambda / 2 \pi) \int_{\partial \Omega}\left\{\sigma_{2}(Q)-\sigma_{1}(Q)\right\} d Q
$$

we obtain (2.3) for the special case $\sigma_{2}(Q) \geq \sigma_{1}(Q)$.

Similarly, we derive (2.4) as follows. Using formula (2.2) first for the function $\sigma_{1}(Q)$, then for the function $\sigma_{2}(Q)$ and subtracting the second one from the first, we find, for any $a<\mu_{1}\left(\sigma_{2}\right)$, that

$$
\begin{array}{r}
2 \int_{a}^{+\infty} \frac{d \Phi(\lambda)}{(\lambda+P)^{3}}+\psi(P)=\frac{1}{8 \pi P^{3 / 2}} \int_{S}\left\{\sigma_{2}(Q)-\sigma_{1}(Q)\right\} d Q+O\left(\frac{1}{P^{2}}\right) \\
\text { as } P \rightarrow \infty,
\end{array}
$$

and consequently

$$
\int_{a}^{+\infty} \frac{d \Phi(\lambda)}{(\lambda+P)^{3}} \sim \frac{1}{16 \pi P^{3 / 2}} \int_{S}\left\{\sigma_{2}(Q)-\sigma_{1}(Q)\right\} d Q, \quad \text { as } P \rightarrow \infty .
$$

As we have done before, we see that

$$
\Phi(\lambda) \sim\left(\lambda^{3 / 2} / 3 \pi^{2}\right) \int_{S}\left\{\sigma_{2}(Q)-\sigma_{1}(Q)\right\} d Q \quad \text { as } \lambda \rightarrow \infty .
$$

Analogously, one establishes the asymptotic formula

$$
\sum_{\mu_{k}\left(\sigma_{1}\right) \leq \lambda}\left\{\mu_{k}\left(\sigma_{2}\right)-\mu_{k}\left(\sigma_{1}\right)\right\} \sim\left(\lambda^{3 / 2} / 3 \pi^{2}\right) \int_{S}\left\{\sigma_{2}(Q)-\sigma_{1}(Q)\right\} d Q \quad \text { as } \lambda \rightarrow \infty .
$$

On using (2.25) and the fact that as $\lambda \rightarrow \infty$ the functions (2.26) for $\sigma^{*}$ and likewise for $\sigma_{*}$ are asymptotically equal to

$$
\left(\lambda^{3 / 2} / 3 \pi^{2}\right) \int_{S}\left\{\sigma_{2}(Q)-\sigma_{1}(Q)\right\} d Q
$$

we obtain (2.4) for the special case $\sigma_{2}(Q) \geq \sigma_{1}(Q)$.

In order to prove the theorem in the general case it is sufficient to apply the equality

$$
\begin{aligned}
\sum_{\mu_{k}(\sigma) \leq \lambda}\left\{\mu_{k}\left(\sigma_{2}\right)-\mu_{k}\left(\sigma_{1}\right)\right\} & \\
& =\sum_{\mu_{k}(\sigma) \leq \lambda}\left\{\mu_{k}\left(\sigma_{0}\right)-\mu_{k}\left(\sigma_{1}\right)\right\}-\sum_{\mu_{k}(\sigma) \leq \lambda}\left\{\mu_{k}\left(\sigma_{0}\right)-\mu_{k}\left(\sigma_{2}\right)\right\}
\end{aligned}
$$


where $\sigma_{0}(Q)=\max \left\{\sigma_{1}(Q), \sigma_{2}(Q)\right\}$ and we apply the special case of the theorem which we just proved.

\section{Further results}

In this section we establish some results which can be considered as a generalisation of the formulae (2.3) and (2.4). Using Theorem 2 , we easily prove the following theorems:

THEOREM 3. Let the functions $\sigma(Q), \sigma_{1}(Q), \sigma_{2}(Q)$ and the quantity $I=$ $\int_{\partial \Omega}\left\{\sigma_{2}(Q)-\sigma_{1}(Q)\right\} d Q \neq 0$ be the same as in (2.3). Furthermore, on the halfaxis $[a,+\infty)$ let a function $f(\lambda)$ of constant sign be given which is absolutely continuous on each interval $[a, b], b<\infty$; further we assume that the expression $\lambda f^{\prime}(\lambda) / f(\lambda)$ is bounded almost everywhere and $\int_{a}^{+\infty} f(\lambda) d \lambda=\infty$. Then as $\lambda \rightarrow \infty$

$$
\sum_{0<\mu_{k}(\sigma) \leq \lambda} f\left(\mu_{k}(\sigma)\right)\left\{\mu_{k}\left(\sigma_{2}\right)-\mu_{k}\left(\sigma_{1}\right)\right\}=\left\{\frac{I}{2 \pi}+o(1)\right\} \int_{a}^{\lambda} f(t) d t .
$$

THEOREM 4. Let the functions $\sigma(Q), \sigma_{1}(Q), \sigma_{2}(Q)$ and the quantity $J=\int_{S}\left\{\sigma_{2}(Q)-\sigma_{1}(Q)\right\} d Q \neq 0$ be the same as in (2.4). Furthermore, on the half-axis $[a,+\infty)$ let a function $f(\lambda)$ of constant sign be given, which is absolutely continuous on each interval $[a, b], b<\infty$; further we assume that the expression $\lambda f^{\prime}(\lambda) / f(\lambda)$ is bounded almost everywhere and $\int_{a}^{+\infty} \lambda^{1 / 2} f(\lambda) d \lambda=\infty$. Then as $\lambda \rightarrow \infty$

$$
\sum_{0<\mu_{k}(\sigma) \leq \lambda} f\left(\mu_{k}(\sigma)\right)\left\{\mu_{k}\left(\sigma_{2}\right)-\mu_{k}\left(\sigma_{1}\right)\right\}=\left\{J /\left(2 \pi^{2}\right)+o(1)\right\} \int_{a}^{\lambda}|t|^{1 / 2} f(t) d t
$$

PROOF. On setting $\alpha(\lambda)=\sum_{0<\mu_{k}(\sigma) \leq \lambda}\left\{\mu_{k}\left(\sigma_{2}\right)-\mu_{k}\left(\sigma_{1}\right)\right\}$, we deduce for any $a<\mu_{1}(\sigma)$ that

$$
\sum_{0<\mu_{k}(\sigma) \leq \lambda} f\left(\mu_{k}(\sigma)\right)\left\{\mu_{k}\left(\sigma_{2}\right)-\mu_{k}\left(\sigma_{1}\right)\right\}=\int_{a}^{+\infty} f(\lambda) d \alpha(\lambda) .
$$

On inserting (2.3) and (2.4) into (3.3), we get easily the formulae (3.1) and (3.2) respectively.

COROLLARY 1. Assuming that the function $f(\lambda)$ of Theorem 3 has the form

$$
f(\lambda)=\lambda^{m}, m \geq-1
$$


we find as $\lambda \rightarrow \infty$ that

$$
\begin{aligned}
\sum_{0<\mu_{k}(\sigma) \leq \lambda} \mu_{k}^{m}(\sigma)\left\{\mu_{k}\left(\sigma_{2}\right)-\mu_{k}\left(\sigma_{1}\right)\right\} \\
= \begin{cases}(I /(2 \pi(m+1))) \lambda^{m+1}+o\left(\lambda^{m+1}\right), & \text { if } m>-1, \\
(I /(2 \pi)) \ln \lambda+o(\ln \lambda), & \text { if } m=-1 .\end{cases}
\end{aligned}
$$

COROLlaRY 2. Assuming that the function $f(\lambda)$ of Theorem 4 has the form

$$
f(\lambda)=\lambda^{m}, m \geq-3 / 2
$$

we find as $\lambda \rightarrow \infty$ that

$$
\begin{aligned}
\sum_{0<\mu_{k}(\sigma) \leq \lambda} \mu_{k}^{m}(\sigma)\left\{\mu_{k}\left(\sigma_{2}\right)-\mu_{k}\left(\sigma_{1}\right)\right\} & \\
& = \begin{cases}\left(J /\left(2 \pi^{2}\left(m+\frac{3}{2}\right)\right)\right) \lambda^{m+3 / 2}+o\left(\lambda^{m+3 / 2}\right), & \text { if } m>-3 / 2, \\
\left(J /\left(2 \pi^{2}\right)\right) \ln \lambda+o(\ln \lambda), & \text { if } m=-3 / 2 .\end{cases}
\end{aligned}
$$

\section{Discussions and conclusions}

The spectral function $\theta(t)=\sum_{k=1}^{\infty} \exp \left(-t \mu_{k}(\sigma)\right)$, where $\left\{\mu_{k}(\sigma)\right\}_{k=1}^{\infty}$ are the eigenvalues of the Laplacian in $R^{n}, n=2$ or 3 , is studied for a variety of domains. The dependence of $\theta(t)$ on the connectivity of a domain and the third boundary condition is analysed. Particular attention is given to a simply connected bounded domain with a positive function $\sigma$ entering the boundary condition of (1.1).

In this paper we have shown that the formulae (1.6) and (1.7) which have been obtained in [6] and [8] when $\sigma$ is a positive constant can be generalised to the formulae (2.1) and (2.2) respectively when $\sigma$ is a positive function satisfying the Lipschitz condition. We also have shown that the formulae (2.1) and (2.2), together with the Tauberian theorem for Hardy and Littlewood, play an important role in the derivation of the asymptotic formulae (2.3) and (2.4), as well as their generalisations (3.1) and (3.2).

\section{Acknowledgement}

The author would like to express his sincere thanks to the referees for their suggestions and comments. 


\section{References}

[1] R. Courant and D. Hilbert, Methods of Mathematical Physics Vol. 1, Wiley-Interscience, New York (1953).

[2] M. S. P. Eastham, "An eigenvalue problem with the parameter in the boundary condition", Quart. J. Math. Oxford 13 (1962) 304-320.

[3] I. M. Gel'fand and B. M. Levitan, "On a simple identity for eigenvalues of a second order differential operator", Dokl. Akad. Nauk. SSSR 88 (1953) 593-596.

[4] A. Pleijel, "A study of certain Green's functions with applications in the theory of vibrating membranes", Ark. Mat. 2 (1953) 553-569.

[5] __, "On Green's functions and the eigenvalue distribution of the three-dimensional membrane equation", Skandinav. Mat. Konger. XII (1954) 222-240.

[6] B. D. Sleeman and E. M. E. Zayed, "An inverse eigenvalue problem for a general convex domain", J. Math. Anal. Appl. 94 (1983) 78-95.

[7] E. C. Titchmarsh, Eigenfunction expansions associated with second-order differential equations, Vol. 2, Clarendon Press, Oxford (1958).

[8] E. M. E. Zayed, "An inverse eigenvalue problem for a general convex domain: An extension to higher dimensions", J. Math. Anal. Appl. 112 (1985) 455-470. 